\title{
Integración Neural del Pensamiento y la Acción Analizada con Técnicas de Computación en Línea
}

\section{Neural Integration of the Thinking processes and Behavior. On line Computational Analysis}

\author{
Guy Santibañez-H ${ }^{1}$ y Sebastián Loma-Osorio J. ${ }^{2}$
}

\begin{abstract}
Resumen
El estudio de las neuroimágenes ha permitido hacer algunos progresos en el conocimiento de los componentes neurológicos que se detectan con las técnicas de computación en línea. Éstas técnicas permiten mantener bajo control operativo la representación de los procesos en estudio, influir intencionalmente en estos procesos, almacenarlos y observar sus cambios. De esta manera, el desarrollo temporal de los fenómenos estudiados es directamente accesible al investigador.

Este trabajo presenta el estado actual del estudio sobre la habilidad del sistema nervioso de reflejar diversos aspectos de los medios con los cuales los seres vivos interactúan y operan predictivamente sobre ellos. Es decir, se refiere a cuestiones básicas de los procesos gnósticos, presentado evidencia de que en su génesis y desarrollo la actividad del sistema nervioso es imprescindible, ya que sin él no podrían producirse fenómenos subjetivos. El análisis de los procesos de memoria, atención selectiva, así como el estudio de tareas complejas como el cálculo aritmético o la ejecución del Test de Raven muestran la activación de diversas áreas corticales. Así, los cálculos aritméticos o las simples estimaciones envuelven una mayor extensión de las áreas parietales que de las áreas frontales, mientras que las imágenes cerebrales durante la ejecución del Test de Raven muestran la activación de conjuntos neuronales que son puestos en acción tanto por configuraciones estimulantes concretas, como por configuraciones estimulantes abstractas.
\end{abstract}

\begin{abstract}
The study of neuroimages has led to some progress in the knowledge of the neurological components that can be detected by on line computational techniques. With these techniques the representation of the studied processes can be kept under operational control, the processes can be influenced intentionally, they can be stored and their changes can be observed. In this manner, the temporal development of the phenomena under study is directly accessible to the investigator.
\end{abstract}

1 Profesor Titular Departamento de Psicología, Universidad de Chile. Email: gsantiba@uchile.cl

2 Psicólogo, Universidad de Chile 
This paper shows the present state of the study on the ability of the nervous system to reflect different aspects of the means through which living beings interact and operate predicatively on them. Thus, the study refers to basic questions of gnostic processes, providing evidence that in their genesis and development, the activity of the nervous system is indispensable, as subjective phenomena could not occur without it. The analysis of memory processes, selective attention, as well as the study of complex tasks, such as arithmetical calculation or the performance of the Raven Test, show the activation of diverse cortical areas. Thus, arithmetical calculations or simple estimations involve a greater extension parietal areas than frontal ones, while during the execution of the Raven Test, brain images show an activation of neuronal sets, both by concrete as well as by abstract stimulating configurations.

\section{Introducción}

Hay muchas opiniones sobre lo que es o se entiende por "conocimiento". Sin embargo, recurriendo a la más modesta y simple aproximación al conocimiento hay una condición que de una manera casi ingenua se hace presente. Conocer es un proceso que ocurre en un tiempo variable. En términos generales, es una relación transitoria entre una entidad viva que conoce - conociente y un objeto de conocimiento involucrado en el proceso gnóstico, entidad conocible. Ambas entidades están en interacción con uno o varios "medios" que las condicionan. De esta manera, es indispensable considerar el condicionamiento histórico, evolutivo y el desarrollo, tanto de la entidad conociente como de la entidad conocible.

La Gnoseogénesis (Santibáñez-H, 2001), tiene una exigencia básica: interacción entre la entidad cognoscente y el objeto de conocer. Esta interacción puede presentar diversas configuraciones fenomenológicas, pero sin ella no hay génesis de conocimiento. La interacción gnóstica tiene que ser capaz de generar una representación subjetiva del objeto conocible. Hecho que permite reconocer sucesivamente el objeto de conocimiento, ubicarlo en sus características espacio-temporales y, de este modo, percibir el incremento del cuanto cognitivo.
El conocimiento empieza por adecuar la actividad perceptual al objeto. La percepción permite evaluar la significación representacional a medida que el proceso gnóstico tiene lugar. Toda percepción inicial refleja sólo algunas propiedades del objeto, estas son las más sobresalientes, es decir, el objeto en esta fase del proceso gnóstico es percibido como una abstracción (Ilyenkov, 1982).

El proceso gnóstico enriquece la representación perceptual del conocible, hecho que permite considerar la percepción como un proceso plástico, de aprendizaje. Esto es claramente comprensible si se considera que cada proceso gnóstico esta modulado por un estado emocional (motivación), el cual tiene un efecto perceptual que puede ser positivo o negativo: facilitar la percepción o suprimirla. En la medida que la respuesta es positiva, la representación del conocible se va enriqueciendo. Podría considerarse a la subjetivación del conocible como un refuerzo, cuya categoría dependerá de la reacción emotiva que activa la respuesta perceptiva. A medida que el conocible es explorado, no sólo perceptualmente sino también instrumentalmente, el fenómeno perceptual se convierte en un fenómeno gnóstico. Hecho que modifica sustancialmente la relación gnóstica. La representación subjetiva del conocible puede ser evocada sin la presencia del objeto. La percepción adquiere de esta manera una diná- 
mica que refleja los cambios experimentados por el objeto. Desde esta perspectiva, la variación perceptual y representacional forma parte del conocimiento. Se puede caracterizar el conocimiento como una modificación perceptual inducida por la experiencia, empírica o epistémico, que confiere la habilidad de modificar el comportamiento del objeto, de predecir los cambios que experimenta y, en la etapa máxima de desarrollo cognitivo, la posibilidad de producir el objeto.

El conocimiento tiene diversas fases que envuelven sucesivamente diferentes "porciones gnósticas" que van desde la habilidad de reconocer el objeto gnóstico hasta la habilidad de reproducirlo. Una etapa intermedia confiere la habilidad de predecir anticipatoriamente el comportamiento el objeto, fenómeno o proceso frente a determinadas influencias que pueden operar, aunque el conocimiento no haya alcanzado su pleno desarrollo.

El Homo sapiens ha sido capaz de desarrollar una serie de habilidades que permiten influir positiva o negativamente sobre los fenómenos vivos, pero aún no está en posición de los medios que permitan crear vida.

Las habilidades adaptativas de las especies implican un conocimiento de las exigencias que demanda el "medio" a los individuos. La sobrevivencia de las especias se debe a sus habilidades adaptativas, a las condiciones ecológicas de los medios en los cuales habitan. Esta adaptación tiene dos fuentes. Las reacciones genéticamente determinadas y las adquiridas por aprendizajes. Es decir, las pertenecientes a la dotación genética y las que se generan en la habilidad de adquirir hábitos que son propios de los individuos.

La génesis del conocimiento no es un mérito exclusivo del Homo sapiens, es una condición funcional de la materia viva.

La historia del conocimiento empieza mucho antes de la existencia de los seres huma- nos. En su etapa primaria, el conocimiento estuvo dirigido a satisfacer las demandas de la materia viva, a resolver los problemas que dificultan la subsistencia. El objeto de conocimiento evoluciona desde este plano, pragmático y concreto, a una condición epistémica. Es decir, se convierte en un producto que debe responder a exigencias que son capaces de asegurar su validez, permitiendo que esta actividad sea capaz de reflejar realmente los acontecimientos a los cuales se refiere.

Conocer es un proceso que envuelve dos instrumentos psicológicos: allí se integran procesos subjetivos (imágenes, ideas) con la habilidad de los seres vivos para operar sobre el medio externo, el comportamiento. El conocimiento se genera y se acumula en el cerebro.

Es interesante destacar que en la medida que los fenómenos gnósticos se van acumulando en los compartimientos cerebrales pertinentes, pueden ser puestos en ejercicio sin activar los fenómenos subjetivos que han contribuido a su génesis. De esta manera, las reacciones gnósticas pueden ser puestas en acción de una manera automática, es decir, sin gatilladores ni acompañadas de los aspectos subjetivos que eventualmente participaron en el proceso de adquisición gnóstica.

Los fenómenos subjetivos - automáticos o conscientes - configuran un medios que hemos denominado el "medio subjetivo" (Santibáñez-H, G., 1991) y que en analogía a los medios interno y externo, este es un medio virtual que está en interacción con el sistema neuroendocrino y es entonces capaz de activar este sistema, tal como sucede con el medio externo y el medio interno (Santibáñez-H, G., 2002).

El conocimiento es característico para cada especie. La evolución de las habilidades adaptativas está basada ampliamente en la actividad integrativa del cerebro. 
Gnoseogénesis es el proceso que lleva a un ser viviente a adquirir conocimientos. En términos generales, el conocimiento se hace conociendo. El conocimiento es fundamentalmente un procesamiento de información de los acontecimientos en los cuales los individuos de una especie están interesados.

Al analizar la gnoseogénesis - la interacción "conociente-conocido" - encontramos que este proceso tiene lugar en condiciones espacio-temporales definidas, en una condición motivacional específica que afecta a quien conoce.

Hay que distinguir dos posibles condiciones motivacionales. Si la gnoseogénesis es mantenida por un fuerte impulso motivante, nos encontramos frente a un modo intencional de relación. En cambio, si el proceso perceptual se produce sin que la persona preste atención específicamente al fenómeno que llegará a convertirse en un elemento conocido, estamos frente a un modo incidental de relación gnóstica.

En síntesis, la gnoseogénesis intencional es un proceso de aprendizaje que puede acontecer de modo intencional o incidental, es una especie de fase preparatoria que lleva a la obtención del conocimiento buscado. La obtención del conocimiento pone fin a la búsqueda, constituye un análogo a la fase consumatoria.

En el análisis del conocimiento, una cuestión importante es la consideración de la naturaleza del conocimiento como fenómeno. En primer término debe considerar la cuestión ¿Cuál es el órgano responsable del fenómeno de conocimiento?

Esta pregunta, en algunos de sus aspectos, la responderemos en el desarrollo del presente trabajo.

\section{La Computación Mental en Línea}

El proceso adaptativo es una forma de interacción del individuo y sus medios. La adaptación al medio externo implica una coordinación de los procesos subjetivos, virtuales y las habilidades operativas del sujeto sobre el medio.

Este análisis se refiere a los resultados obtenidos por diversos autores al registrar las «neuroimágenes» cerebrales con una técnica conocida como "computación mental en línea « («mental computation on line»).

Esta técnica, permite observar el desarrollo de la actividad neural que genera el curso de los procesos subjetivos, como por ejemplo, lo que sucede al pensar en un problema, al operar sobre la representación subjetiva del problema, al memorizar, el resultado de estas operaciones y el almacenamiento del curso de ellas.

Un ejemplo ilustrativo es el cálculo mental, digamos una multiplicación de dos números de dos dígitos cada uno. Experimentos directos y la simulación de sus resultados en computadoras permiten hacer explícito el proceso subjetivo que subyace al cálculo. En primer lugar hay una activación de la memoria de trabajo que permita tanto guardar las cifras como los resultados parciales de la operación. Luego se van separando las cifras y obteniendo resultados parciales. Se requiere retener los resultados parciales y luego sumarlos. Esta operación muestra claramente la importancia de la memoria de trabajo, la habilidad de percibir selectivamente los resultados parciales, el uso de las técnicas operatorias (multiplicar, sumar) y la habilidad de saltar desde la actividad operatoria a las cifras retenidas. Hay en este procesamiento tres fenómenos psicológicos envueltos: los procesos atencionales, la disponibilidad subjetiva de los datos perceptuales rememorados y los contenidos de la memoria de trabajo. El pensamiento «corre» para llenar las demandas del problema, lo cual implica una estrategia para alcanzar un determinado objetivo que resulta de cumplir algunos objetivos parciales que conducen al resultado buscado. 
Un procesamiento análogo es empleado en la resolución del Test Matrices Progresivas de Raven, un test de inteligencia no verbal diseñado para medir la fluidez del razonamiento. Este análisis implica descomponer el proceso estudiado en diferentes aspectos y luego relacionar cada una de sus componentes con el substrato neura! responsable.

Smith y Jonides (2002) sintetizan los componentes de la computación en línea señalando los siguientes componentes «(1) La memoria de trabajo como un artefacto de depósito, (2) actualización de la memoria de trabajo, (3) atención selectiva, (4) cambios atencionales, (5) procesos específicos de las tareas complejas como operaciones aritméticas, manipulación subjetiva de configuraciones especiales, comparación de percepciones visuales, inducción de hipótesis de configuraciones comunes y diferentes etc.».

Para investigar la actividad neuronal, la mayor parte de los trabajos utilizan neuroimágenes tanto la tomografía de emisión de positrones (PET), como la resonancia magnética funcional (fMRI). Las imágenes son obtenidas mientras los sujetos desarrollan diversas tareas cognitivas y durante las pruebas de control. Luego estas imágenes son comparadas para determinar que áreas cambian sus configuraciones funcionales durante el ejercicio. Es también importante relacionar el resultado de las técnicas mencionadas con las lesiones que ciertos pacientes puedan presentar en las zonas de donde se han obtenido las imágenes. (Posner y Raidle, 1944).

\section{Memorización de Trabajo y Almacenamiento.}

Con respecto al almacenamiento y a la actualización de la memoria de trabajo, podría pensarse que los contenidos de la memoria pueden variar, sin que el almacenamiento sea afectado por la variedad informacional. Smith y Jonides (1999) han mostrado que esto no es así. Registrando neuronas de la corteza prefrontal dorso lateral de primates, encontraron neuronas que responden únicamente a la información espacial, mientras que otras neuronas responden únicamente cuando la percepción visual es requerida y debe ser mantenida un breve periodo. Estudios realizados en humanos con PET, encontraron diferentes padrones de activación dependiendo de sí el material almacenado por un breve periodo es espacial, un objeto visible, o una información verbal.

La memoria de trabajo verbal se pone en juego cuando se debe retener un corto tiempo una serie de números, letras, palabras u otros elementos verbales. Por ejemplo leer una serie de números como 27728618, dejar de mirar el texto donde está escrita la serie y después de 5 segundos repetir la serie. Personas sometidas a ésta prueba cuentan que durante los 5 segundos «repiten» mentalmente la serie en cuestión, es decir, durante el tiempo que deben retener la información. Este lenguaje implícito favorece la retención de los dígitos: mientras más rápida es la repetición, mejor resultados se tiene en la prueba. Las conclusiones que se han obtenido en ésta línea de investigación indican que la memoria de trabajo verbal usa una codificación del sonido de los elementos a retener (codificación fonológica) y que esas representaciones fonológicas pueden mantenerse por la repetición del proceso que conforma el lenguaje interno (Baddeley, 1992).

Shallice (1988), describió el «Síndrome de memoria de corta duración» (Short-term memory syndrome) desencadenado por un daño cerebral que produce un deterioro de la memoria de corta duración cuyo déficit primario es la incapacidad de almacenar información verbal durante un par de segundos. En contrate, los pacientes pueden ser capaces de retener, por ejemplo, largas listas de palabras durante un largo tiempo, lo cual indica que la «memoria de larga duración» no ha sido afectada. Este tipo de memoria parece no al- 
terarse con lesiones de la región ubicada en la circunvolución supramarginal del hemisferio izquierdo. Esta zona está frecuentemente dañada en pacientes afectados por el síndrome de la memoria de corta duración.

Para dilucidar este problema, se han llevado a cabo experimentos utilizando «Tests de reconocimiento de Ítems» y controlando la actividad cerebral mediante PET. En uno de estos trabajos se controló el almacenamiento de información en dos condiciones. Estas eran diferentes en cuanto a la cantidad de información. En ambas condiciones, en cada ensayo, un conjunto de cuatro letras fueron presentados brevemente (200 mseg), seguida de una pausa de $3000 \mathrm{mseg}$, al término de la cual se mostraba la letra-test. El sujeto debía decidir tan rápidamente como fuere posible si la letra-test correspondía a algunas de las mostradas en el conjunto. Para ello debía apretar una de dos palancas puestas a su disposición. La mayor diferencia entre las dos condiciones, consistía en que las letras presentadas al grupo de control eran todas iguales, mientras que el grupo experimental eran cuatro letras diferentes. El grupo experimental debía almacenar cuatro diferentes ítems, mientras que el grupo de control debía almacenar un solo ítem. La comparación entre las dos condiciones permitía operar selectivamente la memoria de trabajo verbal aislada. Las imágenes obtenidas en ambas condiciones consistían en un conjunto de datos que mostraban un cambio relativo en el flujo sanguíneo, particularmente en una imagen horizontal de la corteza. El aumento del flujo sanguíneo aumenta en paralelo al incremento de la actividad neural. De esta manera las imágenes del cerebro revelan cuales zonas son más activas.

Cuando la imagen de control fue sustraída de las figuras experimentales, un cierto número de regiones aparecieron significativamente activadas. Es de presumir que esas zonas participen en el almacenamiento y en la repetición en la memoria verbal de trabajo. Es importante tener presente que una de esas zonas de la corteza parietal posterior del hemisferio izquierdo, es la misma región envuelta en la conformación del «síndrome de memoria de corta duración» (Área 40 de Brodmann). Hay, sin embargo, también otras regiones activadas en este proceso: las cuales incluyen la región anterior del hemisferio izquierdo comprometidos en la producción y planificación del lenguaje (área de Broca - B44), el área premotora (BA6) y el área motora suplementaria o A.M.S (también ubicada en BA6. Dado que estas áreas están relacionadas en el lenguaje pueden ser también responsables del lenguaje implícito (repetición).

Estos resultados han sido confirmados por diversos autores dando un sólido respaldo al conocimiento del hecho que la zona posterior almacena y la anterior es responsable de la repetición. Ambas en la región parietal.

La tarea de reconocer debe considerarse un requisito para poner al día la memoria de trabajo en cada ensayo, los antiguos objetivos mnémicos tienen que ser abandonados y reemplazados por un nuevo conjunto. Esta renovación de los recuerdos puede tener varios aspectos. Consideremos el caso en el cual la representación mnémica debe ser, solamente modificada en vez de reemplazada. En este caso puede que no exista necesariamente la participación de nuevas estructuras cerebrales, sino que el proceso de "poner al día» implique solamente de agregar nuevos elementos a los ya almacenados. Por ello es conveniente trabajar con un tipo de «memoria de trabajo» que claramente requiera extensos renovaciones modificatorias.

Awh et al., (1996) utilizando un modelo de tarea que consistía en presentar a sus sujetos una secuencia continua de letras, quienes debían decidir si cada letra de la serie era idéntica a una letra presentada dos letras detrás de ella, o dos letras delante de ella. Esta tarea requiere un almacenamiento complicado ya que implica operar con dos o tres 
letras (las dos últimas letras más la letra en cuestión). Además requiere constantemente la puesta al día (renovación) de la representación mnémica, de manera tal que la letra incorporada primero al conjunto sea abandonada y la letra corriente sea agregada.

Emergen aquí algunas cuestiones de interés: ¿Las imágenes cerebrales activadas por las tareas mencionadas indican una participación de los conjuntos neuronales mencionados?

¿Emergen activaciones de nuevas regiones neuronales que lleven acabo está tarea?

Al comparar las activaciones asociadas en los registros de control con las activaciones observadas durante la ejecución de las tareas, resulta que las imágenes experimentales inducen activación en el hemisferio izquierdo en la región parietal posterior, la cual presumiblemente participa en el almacenamiento. También se activa la región anterior del hemisferio izquierdo, la cual puede considerarse como el locus del lenguaje - discurso - interno (área de Broca, área premotora y área motora suplementaria). Estas regiones no se activan en tareas estrictamente de memorización como reconocimiento de ítems.

Áreas homologas del hemisferio derecho se activan en determinadas circunstancias, lo cual podría indicar que la tarea es más difícil al ser comparada con el reconocimiento de ítems.

Smith y Jonides (1999), han mostrado que la corteza prefrontal dorso lateral (DLPFC Área BA 46) interviene también cuando las tareas a las cuales se someten los sujetos sobrepasan el reconocimiento de Ítems e implican parcialmente una actualización (o puesta al día) o algún otro proceso ejecutivo.

\section{Atención Selectiva: Activación e Inhibición}

La atención selectiva es una función presente cada vez que es necesario seleccionar una información de un conjunto informacional complejo. Esta función es importante en el conocimiento de los procesos de «pensar fluido» (on line computation) (Baddeley 1992).

Smith y Jonides (2002) sugieren que la atención selectiva tiene dos grandes componentes: «activación» de procesos que favorecen la «atención» dirigida a las informaciones relevantes e «inhibición» de la atención a las informaciones irrelevantes.

Esta situación, ha sido estudiada en el paradigma comportamental, tarea de Stroop, donde la información de color y la información verbal, a veces compiten y a veces colaboran. En esta prueba los sujetos son enfrentados con el nombre de colores que están escritos con tintas de colores. Si el nombre del color está escrito con la tinta que corresponda al nombre escrito, la reacción es rápida, pero si el nombre del color no corresponde al color de la tinta con la cual está escrito, la reacción tiene una latencia mayor. Por ejemplo si el nombre «rojo» esta escrito con tinta «roja», la reacción es rápida. En cambio si la palabra «rojo» está escrita con tinta «azul» la reacción se alarga. En el primer caso el fenómeno cromático y el fenómeno verbal son «compatibles», mientras que en el segundo caso son «incompatibles». En la situación de compatibilidad hay una suma de excitaciones. En el caso de la «incompatibilidad» hay una interacción de actividades inhibitorias y excitatorias, que interfiere con el desarrollo rápido de la tarea.

Hay una serie de otras situaciones comportamentales que dependen de una resolución de interferencias del tipo descrito más arriba. Se mencionan, por ejemplo, el efecto de la compatibilidad estimulo-respuesta: estímulos que naturalmente son compatibles con una respuesta se asocian más rápidamente y más precisamente que la configuración estimulo-respuesta incompatible (Kornblum et al.1990). Eriksen y Eriksen 
(1974) observaron una diferencia en la respuesta que un determinado estimulo activa si es administrado sólo después de haber sido asociado a ella. Esta respuesta se deteriora si él estimulo es administrado en una configuración estimulante en la cual está rodeado de estímulos que habían sido previamente asociados a respuestas diferentes.

En estas tareas hay un rasgo común: la concurrencia de dos o más fuentes informacionales que son capaces de activar la misma respuesta o como dicen ciertos autores que compiten por activar una respuesta. Las bases neurales de estos fenómenos de «excitación-inhibición» atencional han sido estudiadas en Homo sapiens con técnicas productoras de neuroimágenes (PET-fMRl). Especialmente la tarea de Stroop ha sido analizada numerosas veces. Aunque hay variaciones individuales de las regiones cerebrales que participan en la génesis de estos fenómenos, dos amplias zonas de la corteza frontal parecen estar comprometidas al comparar la situación de condiciones compatibles e incompatibles: la corteza cingulada anterior y la corteza prefrontal dorso lateral.

Con el objeto de determinar las áreas comunes que se activan en todas las tares de «excitación-inhibición» atencional, Jonides et al. (2002) integraron quince publicaciones que se referían a los resultados obtenidos con técnicas de neuroimágenes, incluidas las tareas antes mencionadas. El análisis fue centrado en las áreas de Brodmann donde se encontraron más bien escasos compromisos neuronales. Sin embargo la región cingulada anterior tuvo preeminencia entre ellas, BA24 tuvo significativa presencia en el hemisferio izquierdo, y BA 32 se activó en ambos hemisferios. Un conjunto neuronal significativo situado en el hemisferio izquierdo BA6, una región que se mezcla con la corteza cingulada anterior, además de un grupo neuronal Significativo en la corteza frontal dorso-lateral.
La unidad «activación-inhibición» atencional afecta también la memoria de trabajo. En este caso, una asociación previa entre un estímulo y una reacción interfiere con la codificación y la rememoración (recuerdo) de una nueva asociación. Jonides et al. (1998) han abordado el problema registrando imágenes con PET en dos condiciones críticas. En la condición de «conflictividad baja» un conjunto de letras, una serie de cuatro letras fue presentado rápidamente y luego de un retardo de 3 segundos siguió una letra-test. Los participantes debían decidir tan rápidamente como fuera posible si la letra-test estaba en la serie presentada. Es importante señalar que los test de ítems negativos requerían de una no-respuesta. Sin embargo esto no ocurrió en el último ensayo. Esta tarea no produjo ningún conflicto de tipo cognitivo.

Un simple cambio en algunos de los ítems puede traer cambios del equilibrio de activación-inhibición atencional. En la condición «altamente conflictiva» la mitad de los test negativos habían aparecido en la serie de los ítems-objetivos. Debido a que en la condición anterior esto «recientes negativos» habían sido ensayados unos pocos segundos antes, necesariamente debía encontrarse en un estado de activación y de familiaridad.

De esta segunda situación emergen varios elementos contradictorios: la transferencia negativa de la experiencia previa, la familiaridad de la prueba misma facilita la comisión de errores y la representación contiene una señal, indicando que es una parte del conjunto de pruebas donde la emisión de una respuesta, era correcta. Estos conflictos son capaces de influir sobre el equilibrio «excitatorio-inhibitorio» de los procesos atencionales, inhibiendo la expresión de procesos irrelevantes de familiaridad y activando selectivamente los procesos que examinan las señales relevantes. La predicción es que únicamente los conflictos internos activarían la atención y su inhibición. Esta predicción se apoya en que el compor- 
tamiento de los participantes muestra un tiempo más largo para responder correctamente a las «negativas recientes» que a las negativas no recientes. Las neuroimágenes son de gran interés. La comparación crítica del PET entre condiciones de alta y baja intensidad conflictiva revela que la parte ventro-lateral de la corteza prefontal izquierda, BA45 era el único foco de activación. Esto implica que esta área está envuelta en la regulación «excitatoria-inhibitoria» de la atención. Ella no está incluida entre las áreas que participan en la integración de resultados, antes mencionadas realizada por Jonides et al. (2002).

Resultados similares fueron obtenidos en un experimento de seguimiento ( $\mathrm{D}^{\prime}$ Esposito et al., 1999), en los cuales se empleó fMRI en un análisis de ensayos aislados. En estos experimentos se consideró la condición «altamente conflictiva» y se hizo la comparación critica entre «no» ensayos cuyas pruebas se habían hecho recientemente (negativos recientes) y «no» ensayos cuyas pruebas eran más antiguos (negativos no recientes). En el hemisferio izquierdo, en la región ventro-lateral prefrontal (BA45) había mayor activación para los recientes negativas que para los «no» recientes negativos.

Otros experimentos parecen confirmar la idea de atribuir a (BA45) del hemisferio izquierdo de estar comprometido en la regulación «excitatoria- inhibitoria» de la atención. Thompson-Schill, et al. (1999) examinaron el contraste entre condiciones de bajo y alto conflicto en un paciente que tenía dañada la región media e inferior del lóbulo prefrontal, incluyendo la región prefrontal ventro-lateral del hemisferio izquierdo. Al compararlo con un grupo de control, el cual tenían lesiones que no incluían a (BA45-46), no encontraron nada especial en la ejecución de tareas de baja conflictividad, pero sí marcadas alteraciones de las respuestas en los ensayos con recientes negativas en condiciones altamente conflictivas, donde se incluía tanto alteraciones espaciales y temporales. Esta alteración resultaba de la exageración significativa de las diferencias entre recientes negativas y no recientes negativas, lo cual coincidía con los déficit tanto excitatorios como inhibitorios de la atención.

\section{Cambio de Foco Atencional: Conmutación Atencional}

Otro problema interesante es el cambio del foco atencional. Este proceso envuelve variaciones de la modulación del equilibrio «excitatorio- inhibitorio» atencional. Sin embargo hay en este proceso de conmutación atencional, un fenómeno que involucra al «conmutador». Desde este punto de vista la conmutación atencional puede ser el resultado de la actividad de estructuras neurales específicas, funcionalmente separados de las que regulan la atención. Sin embargo nociones como atención selectiva y conmutación atencional pueden sugerir la existencia de substratos neuronales comunes o interdependencia de ambas funciones. Se puede suponer que aún existiendo las mismas estructuras neuronales la conmutación implique solamente un ajuste del equilibrio «excitatorio inhibitorio» de las estructuras envueltas.

Probablemente la conmutación atencional tiene diferentes substratos neurales dependiendo de la configuración de ésta conmutación: puede haber cambios atencionales sin cambiar el objeto que se considere y también cambios atencionales con cambio de objeto. Esta última situación implica el ir y venir de un objeto a otro, lo cual compromete a dos reacciones atencionales.

Los cambios de atributos de un mismo objeto es una prueba que se usa para evaluar los pacientes con daño frontal. Se usa una prueba, el Test de Selección de Cartas de Wisconsin (Wisconsin Card Sort Task). Los sujetos reciben una serie de cartas, cada una de las cuales contiene objetos que varían en cuatro atributos: color, forma, número, y 
matices. Los sujetos deben ordenar las cartas en uno de cuatro montones, según la característica que se elija para que el sujeto las clasifique. Si el color rojo es elegido, deberán ordenarse en un montón las rojas, en otro las verdes etc. Las personas reciben retroalimentación sobre su elección que efectivamente se refiere al atributo elegido. Luego inesperadamente se cambia de atributo selectivo y las personas deben determinar cual es el atributo conmutado. Esta demanda requiere un cambio de atención desde un atributo a otro. Los pacientes con daño frontal tienen muchas dificultades para llevar a cabo estas tareas en comparación con otros pacientes neurológicos, la alteración comportamental consiste en que ellos mantienen la elección del objeto sin considerar que estos no poseen el atributo relevante. Estos resultados sugieren que la conmutación atencional es un fenómeno más complejo que el atender selectivamente, tarea que pueden realizar pacientes frontales y que la conmutación atencional es una función controlada en la corteza prefrontal.

Para determinar si hay un mecanismo específico de los fenómenos de conmutación atencional es interesante considerar los estudios de Pollman et al, (2000) usando técnicas de fMRI. La tarea a la cual han sido sometidos los sujetos, ha sido denominada «tarea de la explosión» («pop-out task»\}. Esta prueba consiste en una secuencia de ensayos, donde hay una cierta cantidad de cuadrados de los cuales uno es diferente de los demás en algún aspecto crítico, particular. Este evento fuera de serie es un «objetivo», el resto de los cuadrados son los «distractores». Por ejemplo, en algunos ensayos los distractores son verdes, mientras que el objetivo es azul o bien, los distractores se mueven hacia la izquierda, mientras que el «objetivo» tiene un movimiento sinusoidal. En estas circunstancias la aparición del objetivo en escena parece explotar y es prontamente detectado. Hay que notar que la detección del objetivo es más difícil cuando su dimensión relevan- te cambia de ensayo en ensayo (color-movimiento) que cuando permanece constante. Esta diferencia ha sido conceptualizada como el «costo de conmutación».

Pollman et al., (2000) han registrado las imágenes mientras los sujetos llevaban a cabo la tarea y observaron que los ensayos en los cuales la dimensión relevante cambiaba, había un incremento de la activación en varias regiones de la corteza prefrontal de ambos hemisferios, incluyendo la corteza frontopolar del hemisferio izquierdo (BA10) y el lóbulo frontal inferior (BA47), el lóbulo frontal del hemisferio derecho (BA11) y la región cingulada. Numerosas áreas no frontales se activaron también niveles superiores del área parietal y temporal, incluyendo regiones extraestriadas. En lo que respecta a las regiones y a las activaciones frontales, algunas de ellas también aparecían en los estudios de atención selectiva (BA47 izquierda) y la región cingulada anterior. La corteza frontopolar es de mucha significación, probablemente sea esta región responsable de las funciones de conmutación. Los autores del estudio sugieren que la corteza fronto-polar es responsable de la conmutación de atributos y que la activación de las áreas parietales y temporales son responsables de las consecuencias de la redistribución de la atención.

La conmutación atencional entre objetos, en la memoria de trabajo ha sido un tema sometido a estudio. Garavan (1998) ha desarrollado un paradigma comportamental para estudiar el problema. La tarea: una de dos clases de objetos es presentado en una serie de 15-25 ensayos, los cuales deben ser memorizados de un modo acumulativo, llevando la cuenta de las dos clases de objetos. Los objetos pueden ser un cuadrado pequeño (o grande), los sujetos deben ajustar la cuenta su «contador (registro) subjetivo» agregando uno cada vez. Los sujetos señalan que ellos han corregido su cuenta apretando cada vez un botón, lo que desencadena el próximo estimulo. Las personas son controladas 
por intermedio de los registros acumulados al término de las secuencias. Puede suponerse que los contadores forman parte de la memoria de trabajo y que los contadores que permiten mantener ajustadas las cuentas requiere una activación atencional. En consecuencia cuando los cuadros grandes y los pequeños han sido presentado en secuencia, la atención deber ser conmutada desde un contador al otro, lo que no sucede cuando la secuencia es sólo de grandes o pequeños cuadrados, pues el contador permanece sin alteración. La latencia de la respuesta es mayor cuando hay conmutación atencional que cuando no hay tal cambio. Garavan, Ross, Li y Stein (2000) usando la tecnología fMRI, estudiaron las conmutaciones atencionales, comparando las imágenes producidas, ordenadas en bloque de ensayos que contenían un bajo, un medio o un alto número de conmutaciones atencionales en las secuencias. Garavan et al., (2000) buscaron las regiones que incrementaban su activación en la medida que el número de conmutaciones aumentaba. Encontraron varias regiones que presentaban este aumento en la corteza prefrontal, incluyendo el lóbulo mediano frontal del hemisferio derecho (BA10) el lóbulo prefrontal inferior (BA9-6) en ambos hemisferios y el lóbulo cingulado anterior. Tómese en consideración que hay una activación de la región frontal anterior (BA10) situada por delante (anteriormente) de la región dorso lateral de la corteza prefrontal. Los autores mencionados han encontrado aumento de la actividad en varias otras regiones, lo cual los hace concluir que la conmutación atencional es la consecuencia de la activación de circuitos neuronales ampliamente distribuidos.

Smith y Jonides (2002, p, 1389) comentan un estudio de Badre y sus colaboradores quienes usando la técnica fMRI, en vez de promediar las imágenes de bloques de ensayos han utilizado la imagen de cada uno de los ensayos de la secuencia. Los estímulos usados fueron flechas al lado izquierdo versus flechas al lado derecho de la pantalla. Los sujetos debían tener separados las cuentas acumuladas para las dos flechas. Tanto la flecha de la izquierda como la de la derecha podían apuntar tanto hacia arriba como hacia abajo. Si una flecha apuntaba hacia arriba, el contador correspondiente debía sumar una unidad y si apuntaba hacia abajo, debía restar una unidad. Con estos experimentos fue posible para los autores determinar si los sucesivos ensayos demandan la misma operación o sí inducen conmutación operacional, independiente si el ensayo exige una conmuntación del contador (izquierda o derecha) como la conmutación de la operación aritmética (sumar o restar) ó exigen tiempos de ejecución mayores. Los resultados fMRI han mostrado que conmutación en cada ensayo produce activaciones específicas comparados con la línea de base de los ensayos. Estas activaciones son particularmente claras en el área anterior de la corteza prefrontal. Ambos fenómenos, conmutación de los contadores y conmutación de la operación, inducen activaciones de la región fronto-polar del hemisferio derecho (BA10). En concreto, estudios relativamente simples acerca de cambios de la dirección atencional al ser comparados con la conmutación de la atención con equilibrio «excitatorio-inhibitorio» de la atención se observa que estructuras corticales adicionales intervienen en estas conmutaciones, especialmente las regiones anteriores de la corteza prefrontal incluyendo la región dorso lateral de la corteza prefrontal.

$\mathrm{Al}$ examinar los resultados de tareas con el método de computación mental en línea (on line - de registro continuo) se ha concluido que estas tareas incluyen varios componentes, a saber: almacenamiento informacional de los datos de la memoria de trabajo; actualización de la información acumulada en la memoria de trabajo, atención selectiva (equilibrio «excitatorio-inhibitorio» atencional), conmutación atencional, como también «procesos específicos» que cambien de tarea en tarea. 


\section{Los Componentes que Intervienen en Tareas Complejas}

En el análisis de las tareas en las cuales se ha empleado la computación en línea de los procesos subjetivos envueltos, se ha observado que estas tareas concitan la intervención de varios componentes, tales como, el almacenamiento de memorias de trabajo, la adecuación de la memoria de trabajo, la atención selectiva (inhibición-excitación atencional), conmutación atencional; incluyendo los cambios atencionales que son específicos para cada tarea.

Al estudiar tareas de aritmética-mental hay que distinguir aquellas tareas que requieren cálculos reales $(6 \times 8)$ de las tareas que requieren estimaciones o un componente análogo.

Las investigaciones pertinentes indican que la aritmética y la estimación son mediadas por diferentes integraciones neurales. La estimación envuelve más áreas parietales que áreas frontales (Chonchón et al. 1999).

Rickard et al., (2000) sometieron a voluntarios, a un estudio usando fMRI, mientras llevaban a cabo una multiplicación de dos dígitos. Al menos la mitad de las áreas activadas eran áreas «componentes» de otras funciones estudiadas a través de técnicas de computación en línea. Estas áreas incluían, el área de Broca (BA44) la cual participa en el almacenamiento de materiales alfanuméricos y que probablemente interviene en la fijación de productos del lado izquierdo de la ecuación. Se activa también la región anterior de la corteza prefrontal de ambos hemisferios (BAs 9 y 10), las cuales intervienen en fenómenos de atención y de conmutación atencional.

Chonchón et al., (1999) analizando problemas de multiplicación y substracción han encontrado que un 50\% de las áreas activadas corresponden a áreas componentes. En la multiplicación, esos componentes incluyen el área de Broca y el área motora suplementaria, (Área BAs 44 y 6), las cuales están envueltas en et ejercicio de la memoria de trabajo, la región dorso-lateral de la corteza prefrontal (BA46) envuelta en la atención selectiva y posiblemente en la conmutación atencional; y en el lóbulo angulado anterior, que opera en la selectividad atencional.

Prabhakaran et al., (1997) han controlado las imágenes cerebrales durante la ejecución del test de Raven. El autor estudió problemas con diversos grados de complejidad. El problema más sencillo era puramente figurativo. Requería solamente operaciones perceptuales visuales. Mientras que problemas más difíciles, más analíticos, requerían la aplicación de operaciones conceptuales para caracterizar las figuras, generar hipótesis sobre las relaciones que ellas puedan tener. La distinción entre problemas de configuraciones concretas y de configuración abstracta, es importante para el análisis de ciertas tareas. El tratamiento de este problema con técnicas de fMRI revela un cierto número de activaciones frontales bilaterales, las cuales tienen correspondencia con las áreas de las componentes. Estas incluyen el área premotora (BA 6), el área de Broca (BA44), el área de atención selectiva temprana (BA45) y el área de selección selectiva tardía (BA 46 y 9). Muchas de estas áreas estaban lateralizadas en el hemisferio izquierdo, en los estudios de la memoria de trabajo antes examinada que consideraba solamente la memoria verbal, mientras que en los estudios de Raven parecen estar en juego tanto la memoria de trabajo espacial como la visuoobjetal. Este último sistema está parcialmente configurado por regiones del hemisferio derecho como el área promotora y región dorso lateral de la corteza prefrontal (Smith y Jonides, 1999).

Además de las áreas frontales, la comparación entre problemas analíticos y configurativo-perceptuales pone en evidencia la activación de las áreas occipitales, 
parietales y temporales. Algunas de éstas activaciones forman parte de áreas componente como el lóbulo supramarginal izquierdo (BA 40), que al parecer participa en el almacenamiento de información de la memoria de trabajo. Otras de esas áreas pueden estar envueltas en la memoria de trabajo espacial y objetal como BA7 (corteza parietal superior) y BA19 (corteza visual extraestriada).

La computación mental en línea ha sido utilizada para estudiar la memoria de trabajo modulada por la atención selectiva y los cambios atencionales entre procesos de tareas específicas y la actualización de la memoria de trabajo. El análisis de los resultados de estas investigaciones indican que la memoria de trabajo verbal es producida por dos distintas funciones neuronales: un componente de almacenamiento tiene lugar en la región parietal posterior del hemisferio izquierdo y el componente evocado- repetitivo por las regiones frontales del hemisferio izquierdo, que también participan en la planificación y producción del lenguaje. La actualización de la memoria de trabajo Parece ser la consecuencia de incluir la activación de otras regiones, incluida la porción dorsolateral de la corteza prefrontal.

La atención selectiva producida por una relación especifica de la interacción "inhibición-excitación atencional” parece estar regulada por BA24 del hemisferio izquierdo y la BAs32 de ambos hemisferios. Un segundo conjunto neuronal que se activa en estas circunstancias es BA6 del hemisferio izquierdo, región que se mezcla con la corteza cingulada anterior en su extensión ventral medial. Hay además un significativo conjunto neuronal en la región dorso-lateral de la corteza prefrontal derecha.

El examen de las bases neurales de la atención selectiva y de la conmutación atencional ha mostrado que estas funciones envuelven más de una estructura que participan en la resolución de la excitación o inhibición de los procesos atencionales envueltos en la selec- tividad o conmutación atencional. Las estructuras que participan parecen envolver estructuras prefrontales, incluyendo la región cingulada anterior y la región dorso-lateral prefrontal. Otros trabajos parecen mostrar que en la memoria de trabajo, una forma primaria de «excitación-inhibición» atencional, es la consecuencia de la activación en el hemisferio izquierdo en la región prefrontal de la corteza.

En el análisis de la integración neural de las tareas complejas parece emerger que están envueltos los componentes neurales de las tareas simples que hemos resumido más arriba.

En las tareas examinadas envuelven cuatro procesos: memoria de trabajo, adecuación de la memoria de trabajo, procesos de inhibición-excitación en la atención selectiva y conmutación atencional. Distintos substratos neurales han sido encontrados para los diferentes procesos. La memoria de trabajo verbal se integra principalmente en las estructuras del hemisferio izquierdo, comprometiendo la zona parietal posterior (BA40), como también estructuras frontales posteriores (BAs 6 y 44). La adecuación parcial de la memoria de trabajo algunas veces conlleva la activación de la región dorso-lateral de la corteza prefrontal, pero esta inclusión no es muy clara. El equilibrio excitatorioinhibitorio de los procesos atencionales pueden ocurrir con relativo retardo en relación a la activación bilateral de la región dorso-lateral de la corteza frontal y de la región cingulada anterior, mientras que los procesos excitatorios-inhibitorios atencionales pueden ocurrir más temprano en el hemisferio izquierdo en la región ventro-lateral de la corteza frontal (BA45). Los estudios de conmutación atencional fueron menos consistentes en sus resultados. Hay sin embargo alguna evidencia de un claro compromiso de la corteza prefrontal anterior incluyendo la región fronto-polar. 
Las imágenes recogidas en las tareas representativas del pensamiento muestran que esos componentes operan especialmente en los procesos de memoria de trabajo y de selectividad atencional. Estos resultados que integran procesos neurales diferenciados son responsables para las situaciones del racionamiento fluido.

\section{Bases Neuronales de los Procesos Decisionales}

Hay una serie de datos experimentales que describen las bases neuronales envueltas funcionalmente en el proceso decisional de reaccionar a una de las dos alternativas capaces de poner en marcha un determinado movimiento.

Los monos - Macaca-mulata - con los cuales trabajábamos vivían en jaulas que daban a un corredor interno, pero también una salida a un patio común externo. De ésta manera los animales podían observarse recíprocamente. Hacia el patio, cada jaula tenía una puerta que permitía entrar a ella desde ese lado. Las jaulas habrían debido mantenerse cerradas con un candado, pero a falto de éste se usó un adminículo que se podía abrir y cerrar por medio de un sistema de palancas. Una de las monitas, a través de los barrotes, manipulaba el adminículo que era fácil de abrir si se conocía el truco y sobre todo si se tenía acceso cómodo a la cerradura. Para la monita el acceso era difícil e incómodo- Observamos que un día, ella manipulaba enérgicamente. Sólo algunos congéneres prestaban atención. Un par de días después ella seguía manipulando el adminículo. La reactividad social había cambiado: el resto de la población seguía muy atentamente la actividad de la monita. El mismo estímulo determinaba una reacción distinta. Un buen día se sintió un fuerte alboroto en el patio. La monita había abierto la jaula. Una buena parte de los espectadores había decidido trabajar en sus cerraduras. En vista de lo cual serios ........ fueron utilizados. Des- pués de un tiempo había terminado la actividad libertadora: una nueva decisión.

Diversas alternativas de respuesta entre las que hay que elegir una constituye la llamada "capacidad de juzgar" o "tomar decisiones" (Hastie, 2001) esta función del sistema neuroendocrino opera tanto a nivel de procesos subjetivos como comportamentales.

Los trabajos de Newsome, Britten y Movshon (1999) han contribuido a precisar la integración neuronal de estos procesos. Estos autores han registrado neuronas aisladas de una área visual extraestriada ubicada en la circunvolución temporal media del Macaco. Encontraron que la percepción visual del movimiento activaba diversas unidades neuronales. Los animales fueron sometidos a una prueba de discriminación. En la pantalla de un computador había que diferenciar puntos que se movían al azar de otros que se movían coherentemente en una dirección determinada. A medida que cambiaba la proporción de puntos moviéndose coherentemente, en cualquier dirección, la discriminación se hacia más difícil. Los autores mostraron que tanto la agudeza perceptual como la actividad de las neuronas sensibles al movimiento del área media temporal estaban correlacionadas con la fracción de puntos coherentemente en movimiento.

Más aún, la micro estimulación de columnas particulares de las neuronas de la región temporal media, que reaccionan al movimiento direccional de los puntos, favorecen sistemáticamente las decisiones tomadas por los monos en favor de la dirección facilitada por la estimulación de la columna (Salzrnan, Britten y Newsome, 1990) Relaciones similares entre la percepción de diferenciaciones y la activación neuronal de la corteza somato sensorial primaria y secundaria han sido encontradas en monos entrenados a hacer discriminaciones vibro táctiles (Hernández, Zaino y Romo 2000, Romo, Hernández, Zaino, Brody y Lemus, 2000). 
Estos trabajos corroboran que la percepción durante un aprendizaje discriminativo es producida por una activación neuronal ubicada en áreas sensoriales. Muestran la dependencia, la agudeza perceptual del número de neuronas que se activan durante el proceso discriminativo y, que la discriminación se integra en una unidad comportamental gracias a la convergencia, entre otros fenómenos, en neuronas ubicada fuera de las áreas sensitivas primarias y secundarias. La región temporal media tiene conexiones recíprocas con unidades neuronales del campo frontal de los globos oculares y del colículo superior. Estas neuronas controlan los movimientos oculares y la distribución espacial de las fuentes generadoras de estímulo.

Shadlen y Newsome (2000) encontraron actividad neuronal en la corteza parietal y, Kim y Shadlen (1999) en la corteza prefontal. Es interesante señalar que la actividad promedio de las neuronas de las regiones señaladas aumenta cuando el movimiento discriminado se facilita y disminuye cuando se dificulta.

\section{Significado del Conomiento Previamente Adquirido}

Es un hecho conocido que alguien en presencia de una emergencia - por ejemplo un accidente - reaccionará de manera diferente dependiendo de la relación que tenga con la persona accidentada, eligiendo la actitud en virtud de su experiencia histórica con ella o de los principios que haya incorporado $y$, de cómo los ha incorporado.

Platt y Glimcher (1999), y Platt 2002 registraron la actividad de neuronas aisladas del área intraparietal lateral, mientras los monos aprendían a enfocar visualmente uno de los dos «estímulos-objetivos», situados en la periferia de la fuente de estimulación, consistente en la alternancia de dos estímulos de color, en la cual cada color correspondía a cada uno de los estímulos enfocables.
Los puntajes dados por el número de respuestas correctas- enfocar con la mirada al «estímulo-objetivo» adecuado - se mantuvieron variables por la variación de la cantidad del refuerzo o alterando la probabilidad que cada posible cambio de la dirección de la mirada sería señalado en bloques de ensayo. En todo caso los autores encontraron que cuando la señal de color, la localización del objetivo y el movimiento se mantuvieron constantes, la actividad de las neuronas del área intraparietal lateral fue proporcional a la magnitud de respuestas correctas. Similares correlaciones entre la actividad neuronal y los puntajes comportamentales de un particular movimiento ocular, han sido encontradas en la corteza prefrontal (León y Shladen 1999), en el núcleo caudato, (Kawage, Takikawa y Hikosaka, 1990), parte reticulada de la sustancia negra (Handel y Glimcher 2000). En el caso que el animal no reciba una señal específica para elegir una de las alternativas, en ausencia de una clave instruccional, los monos dirigen la mirada al objetivo productor de la mayor cantidad de refuerzo y la actividad de muchas neuronas del área intraparietal lateral (Platt y Glimcher 1999).

Se debe tener en consideración la activación general del sistema neuroendocrino, la cual juega un rol en la toma de decisiones. Las actividades motivacionales están envueltas necesariamente en la resolución de un problema. La decisión es obviamente un problema que un individuo debe resolver. El efecto del refuerzo activa diferentes conjuntos neuronales. Han sido descritas respuestas de los ganglios básales con activación de neuronas dopaminergicas de la parte compacta de la sustancia negra (Substantia nigra pars compacta), las modulaciones de la actividad del núcleo caudato (Shima e Hikosaka 2001), el aumento de la actividad del núcleo acumbens, de la corteza orbito frontal además de extensas áreas de la corteza parietal han sido puestas en evidencia usando técnicos de imágenes de resonancia magnética (Bems, Mc Clure, Pagnoni, Montagne 2001). 
En la actualidad existen una cantidad de datos que permiten estimar como ocurre una toma de decisiones, considerando el sustrato neuronal envuelto en éste proceso. Gold y Shadlen (2000) han aplicado micro estimulación a las neuronas de las áreas frontal ocular, mientras los monos realizaban al azar una tarea de discriminación del movimiento de puntos en una pantalla. Observaron que la micro estimulación producía un movimiento saccadico que desviaba la mirada en la dirección que los animales tomaban cuando no había estimulación. La magnitud de la desviación dependía tanto de la proporción de puntos que se movían coherentemente como del tiempo que el mono había observado el estimulo. Los datos de estos autores sugieren que el plan de desarrollo del movimiento refleja la acumulación gradual de la información del movimiento, como si la decisión y el movimiento en preparación compartieran una organización neural común. Esta organización neural incluye los campos oculares frontales, (Bruce y Goldenberg 1985), los colículos superiores (Muñoz y Wurtz 1995) y la corteza motora primaria que controla los movimientos de la musculatura esquelética (Salina y Romo 1998).

Los experimentos citados no muestran como intervienen las estructuras mnémicas y emóticas. Han sido hechos teniendo en perspectiva solamente la contribución visual o auditiva a la realización de movimientos. De todas maneras es interesante notar que los estados motivacionales y los recuerdos tienen necesariamente una base neuronal que debe tomarse en cuenta si se quiere conocer las bases neuronales de las funciones decisionales.

\section{Bibliografía}

-Awh, E., J. Jonides, E.E. Smith, E. H. Schumacher, R.A. Koeper and S. Katz, 1996, "Dissociation of storage and rehearsal in verbal Working memory: evidence from PET". Psychol. 7:25-31
-BADDEley, A.D., 1992, “Working memory”. Science 255: 556-559

-Bems, G.S., S.M. Mc CluRe, G. Pagnoni AND P.R. Montagne, 2001, "Predictability modulates human brain responses to reward". J. Neurosci. 21:2793-2814

Bruce, C.J. And M.E. Goldenberg, 1985, “Primate frontal eyes field single neurons discharging before saccades". J. Neurophysiol. 53: 443-452.

- Chonchon, F., L. Cohen, P, F. van der Moortele, And S. Dehaene, 1999, "Differential contributions of the left and right inferior parietal lobules to number processing". J. Cogn. Neurosci. 11(6): 617630

-D’Esposito M., B. R. Postle, J. Jonides, AND E.E. SмIтH, 1999, “The neural substrate and temporal dynamics of interference effects in working memory as revealed by event-related functional MRJ". Proc. Natl. Acad. Sci. 96:457-460

-Eriksen, B. A. And C. W. ERiKsen, 1974, "Effects of noise letters upon identification of a target letter in a non search task". Percept Psychophys. 16:143149

-Garavan, H., 1998, "Serial attention within working memory". Memory Cogn. 26:263-276

- Garavan. H. T.J.Ross, S-J. Li, AND E.A. Stein, 2000, "A parametric manipulation of central executive functioning". Cerebral Cortex 10 (6) 585-592

-Gold, J.L. ANd M.N. Shadlen, 2000, "Representation of a decision in developing oculo-motor command". Nature 404: 390-394

- Gray, G.M. ANd W. Singer, 1989, “Stimulus specific neuronal oscillations in orientation columns of cat visual cortex". Proc-Natl Acad. Sci. USA 86:1689-1702. 
-Handel, H.G., P.W. Glimcher, 2000, "Contextual modulation of substantia nigra pars reticulate". J. Neurophysiol. 83:3042-3048

- Hastie, R., 2001, "Problems for judgment and decision making". Annu. Rev. Psychol. 52:653-683

- Hernández, A., A. Zainos and R. Romo, 2000, "Neural correlates of sensory discrimination in the somatosensory cortex". Proc. Nath. Acad. Sci USA, 97:6191-6196

-Ilyenkov, E.V., 1982, "The dialectic of abstract and concret in Marx's Capital". Progress Publishers, Moscow Pp 292

-Jonides, J., E.E. SMith, DC. MARschuetz, R.A. Koeppe, y R.A. Reuter-Lorenz, 1998, "Inhibition in verbal working memory revealed by brain activation". Proc. Natl Acad. Sci. USA 95:8410-8413

- Jonides, J., D., Brade, C. Curtis, S.Thompson SCHILL, AND E.E. SMITH, 2002, “Mechanism of conflict resolution in prefrontal cortex". In D.T. Stuss and P.t. knight, eds. The frontal lobes. Oxford Univ. Press. Oxford.

-Kawagoe, R., Y. TaKiKawa AND O. HiKosaKa, 1998, "Expectation of reward modulates cognitive signals in the basal ganglia". Nat. Neurosci. 1:411-416

-Kim J.N. And M.N. Shadlen, 1999, “Neural correlates of a decision in the dorsolateral prefrontal cortex". Nat. Neuros. 2:176-184

-Kornblum, S. T. Hasbrouce, and A. Osman, 1990, "Dimensional overlap: cognitive basis for stimulus- responses compatibility a model and taxonomy". Psychol. Rev. 97 (2) 253-270

-Leon, M. Y. AND M.N. Shladen, 1999, “Effect of expected reward magnitude on the response of neurous in the dorsal prefrontal cortex of the macaque". Neuron. 24:415-425
- Muñoz, D.P, and R.H. Wurtz, 1995, "Saccade related activity in monkey superior colliculus. 1. Characteristics of burst and building up calls". J. Neurophysiol. 73:2313-2333

- Newsome, W.T., K.H BRitten and J.A. Movshon, 1989, "Neural. Correlate of a perceptual decision" Nature 7:52-54

- Platt, M.L., 2002, “Neural correlates of decisions". Current Opinion in Neurobiology 12:1-8

- Platt, M.L., and P.W. Glimcher, 1999, "Neural correlates of decisions variables in parietal cortex". Nature, 400:233-238

-Pollman, S., A. Y. Dove, D. Von Cramon, AND C. J. Wiggins, 2000, "Event- related fMRI: Comparison of conditions with varying BOLD overlap". Hum. Brain Mapp. 9(1): 26-37

-Posner, M. J. AND M. E RaidLe, 1994, “Images of Mind". Scientific American Library pp. 257

-Prab Ha Karan, V., j. A. L. j. E. Desmond, G.H. Glover, and G. Gabrieli, 1997.

- Rickard, T.C., S.G. Romero, G. Basso, C. Wharton, S. Flitman, and J. Grafman, 2000, "The Calculating Brain: A fMRI study". Neuropsychología, 38: 325-335

-Neural Substracts of. Fluid reasoning.

- Salinas, E., AND R. Romo, 1988, “Conversion of sensory signals into motor command in primary cortex". J. Neurosis. 18: 542545

-SANTibáñ̃ZZ-H, G., 1991, “Subjetivität als die dritte Unwelt". Deutsche Zeitschrift für Philosophie. 5: 464-476

-SAntibÁÑez-H, G. ,2001, “Bases Psicofisiológicas de la Gnoseogénesis". Revista de Psicología Universidad de Chile, Vol. X, Nº 1, Pág. 151-175 
-SAntiBÁÑEz-H, G., 2002, "La Dialéctica de los Procesos Subjetivos". Revista de Psicología Universidad de Chile, Vol. XI, N 1, Pág. 65-90

-Salzman, C.D., K. H. BRitTen, and W.T. Newsome, 1990, "Cortical microestimulation influences perceptual judgments of motion direction". Nature, 346:1714-1717

-Shadlen, M. N. And W.T. Newsome, 2000, "Neural basis of a perceptual decision in parietal cortex (area LIP) of rhesus monkey". J. Neurophysiol. 86:1916-1936

-Shallice, T., 1988, "From Neuropsyl. to Mental Structure". Cambriage Univ. Press. Cambriage

-Shima, Y., and O. Hikosaka, 2001, "Role of tonically active neurons in primate caudate in reward-oriented sacradic eyes movement". J. Neurosci. 21:7804-7814

-SMith, E.E AND J. JonIDES, 1999, “Storage and executive processes in the frontal lobes". Science 283:1657-1661

-Smith, E.E AND J. Jonides, 2002, “Executive Control and Thought. In L. R. Squire and Others". Fundamental Neurosciences, 2Ed. 54:pp 1977-1394

- Thompson- Schill, S. L., J. Jonides, C. Marshuetz, E. E. Smith, M. D’Espocito J. F. Kan, R. T. KNighit, AND D. Swick, 1999, "Impairments in executive control of working memory following prefrontal damage: A case study". Abst. Soc. Neurosci 25:1143

Fecha Recepción Artículo: 10 de marzo 2006 Fecha Evaluación Final: 22 de mayo 2006 